PROCEEDINGS OF THE

AMERICAN MATHEMATICAL SOCIETY

Volume 126, Number 5, May 1998, Pages 1293-1303

S 0002-9939(98)04234-8

\title{
SUBGROUP TRANSITIVITY IN ABELIAN GROUPS
}

\author{
PAUL HILL AND JANE KIRCHNER WEST
}

(Communicated by Ronald M. Solomon)

\begin{abstract}
We generalize an appropriate modification of the classical notion of transitivity in abelian $p$-groups from one that is based on elements to one based on subgroups. We consider those $p$-groups that are transitive in the sense that there is an automorphism of the group that maps one isotype subgroup $H$ onto any other isotype subgroup $H^{\prime}$, unless this is impossible due to the simple reason that either the subgroups are not isomorphic or the quotient groups are not (as valuated groups when endowed with the coset valuation). Slight variations of this are used to define the classes of strongly transitive and strongly U-transitive groups. The latter class is studied in some detail in this paper, and it is shown that every $C_{\Omega}$-group is strongly transitive with respect to countable isotype subgroups.
\end{abstract}

\section{INTRODUCTION}

By a $p$-local group we mean a module over $Z_{p}$, the integers localized at a prime $p$. A torsion $p$-local group is simply a $p$-primary abelian group $G$; this means that each element of $G$ has order a power of the fixed prime $p$. Such a group hereafter will be referred to as a $p$-group.

If $G$ is a $p$-local group, let $p G=\{p x: x \in G\}$. Define $p^{\alpha} G$ inductively, for each ordinal $\alpha$, by letting $p^{\alpha+1} G=p\left(p^{\alpha} G\right)$ and by letting $p^{\beta} G=\bigcap_{\alpha<\beta} p^{\alpha} G$ whenever $\beta$ is a limit. The descending chain of subgroups $\left\{p^{\alpha} G\right\}$ produces a valuation on $G$ defined by $|x|=\alpha$ if $x \in p^{\alpha} G \backslash p^{\alpha+1} G$. Naturally, we define $|x|=\infty$ if $x \in p^{\infty} G=$ $\cap p^{\alpha} G$, where $\alpha$ ranges over all the ordinals. A subgroup $H$ of $G$ is called a valuated subgroup when endowed with the valuation on $G$ (apart from its own valuation). If the valuation on $G$ when restricted to $H$ is identical to the valuation of $H$ itself, then the subgroup $H$ of $G$ is called an isotype subgroup. The latter terminology, perhaps unfortunately, is well established. Two subgroups $A$ and $H$ of $G$ are said to be compatible if, for any $a \in A$ and any $h \in H$, there exists $c \in A \cap H$ for which $|a+c| \geq|a+h|$.

Subgroups $H$ and $H^{\prime}$ of $G$ are said to be equivalent subgroups of $G$ if there is an automorphism of $G$ that maps $H$ onto $H^{\prime}$. Hence, two subgroups are equivalent if they are not only isomorphic as groups but are indistinguishable within the structural framework of the containing group. For example, Sylow's 2nd theorem

Received by the editors May 12, 1996 and, in revised form, October 23, 1996.

1991 Mathematics Subject Classification. Primary 20K10, 20K27; Secondary 20K30, 20E36.

Key words and phrases. Primary groups, subgroup transitivity, equivalent subgroups, automorphisms, totally projective groups.

The first author was supported by NSF grant DMS 92-08199.

(C)1998 American Mathematical Society 
implies that any two Sylow $p$-subgroups of a finite (noncommutative) group $G$ are equivalent. There can now be found in the literature a host of equivalence theorems for abelian groups, but this is a relatively recent development. One of the most important of these is the Main Theorem of [HM], which will be quoted below. Most of the results herein are based on the proof of this theorem.

The notion of transitivity for abelian groups goes back at least to Kaplansky [K]. A $p$-local group $G$ is transitive if, given any two elements $x$ and $y$ in $G$ for which $\left|p^{n} x\right|=\left|p^{n} y\right|$, for each $n<\omega$, there is an automorphism of $G$ that maps $x$ onto $y$. In other words, $G$ is transitive if any two cyclic subgroups of $G$ that are isomorphic as valuated subgroups are, in fact, equivalent subgroups of $G$. The suggestion by Kaplansky in $[\mathrm{K}]$, deleted in the revised edition, that all abelian $p$-groups might be transitive is ancient history. Indeed, there is an example in $[\mathrm{H}]$ of a $p$-group $G$ with two cyclic subgroups $\langle x\rangle$ and $\langle y\rangle$ that are isomorphic as valuated groups, yet the quotient groups $G /\langle x\rangle$ and $G /\langle y\rangle$ are not isomorphic. Since the latter obviously precludes an automorphism of $G$ that maps $x$ onto $y, G$ is not transitive. This example brings into focus the following fundamental fact. In order for two subgroups $H$ and $H^{\prime}$ of $G$ to be equivalent subgroups of $G$, they must satisfy the conditions:

(1) $H \simeq H^{\prime}$, and

(2) $G / H \simeq G / H^{\prime}$.

Moreover, conditions (1) and (2) are necessary when both isomorphisms are interpreted in the valuated sense. In this connection, we should hasten to add that the valuation on the quotient $G / H$, and likewise on $G / H^{\prime}$, is the coset valuation. The value of the coset $g+H$ is denoted by $\|g+H\|$ and is defined by

$$
\|g+H\|=\sup \{|g+h|+1: h \in H\} .
$$

Being mindful of the observation just made, we shall henceforth interpret the isomorphisms in (1) and (2) as isomorphisms in the category of valuated groups; that is, it is understood that these isomorphisms preserve values. Since we intend to restrict our investigation to the case where $H$ and $H^{\prime}$ are isotype subgroups of $G$, the preservation of values becomes a moot issue in regard to (1), but not in regard to condition (2). Incidentally, the consideration of only isotype subgroups $H$ and $H^{\prime}$ is not merely a matter of convenience. It is necessary since it is a requirement of the Main Theorem in $[\mathrm{HM}]$.

All of the preceding has led to the following.

Definition 1. A p-local group $G$ is transitive with respect to isotype subgroups if any two isotype subgroups $H$ and $H^{\prime}$ that satisfy conditions (1) and (2) are equivalent.

Simply and naively stated, $G$ is transitive with respect to subgroups if there is always an automorphism that takes one subgroup onto another, unless there is an obvious reason that precludes such an automorphism. The concept is then formalized by the stipulation that the obvious reasons are the failure of condition (1) or (2). Finally, we remark that it is appropriate to consider transitivity of subgroups as being a generalization of ordinary transitivity, which is the transitivity of elements (or, in the present context, the transitivity of cyclic subgroups). To be sure, the classical concept of transitivity omits (2) and stipulates only condition (1), but condition (1) usually implies (2) in this case primarily because $H$ and $H^{\prime}$ are finite. 
Although we have focused thus far on transitivity, it may be fair to say that our main result is actually Theorem 2 . This theorem asserts that, under reasonable hypotheses, a map can be extended in such a way that it simultaneously lifts a given quotient map.

\section{Preliminaries}

We begin this section by returning, for a moment, to the notion of ordinary transitivity. We shall assume hereafter that the containing group $G$ is an abelian $p$-group. We have already referred to an example of a $p$-group $G$ that is not transitive, but $p$-groups that are not transitive are the exception, not the rule. However, a precise determination of which $p$-groups are transitive has not yet been made. Likewise, we have no complete answer to the question of which $p$-groups are transitive with respect to isotype subgroups. However, we have examples (actually classes of examples) from opposite ends of the structural spectrum of $p$-groups that are, in fact, transitive with respect to isotype subgroups. Nevertheless, transitivity of (isotype) subgroups, unlike the transitivity of elements, appears to be more the exception than the rule, at least when dealing with uncountable $p$-groups. It is well known (see Example 2 below) that all countable $p$-groups are transitive with respect to isotype subgroups.

Example 1. Let $B_{n}=\bigoplus_{\kappa(n)} C\left(p^{n}\right)$ be the coproduct of $\kappa(n)$ copies of the cyclic group of order $p^{n}$, where $\kappa(n)$ is an arbitrary cardinal. Then let $P=\prod_{n<\omega} B_{n}$ denote the product of the $B_{n}$ 's. Finally, let $G$ be the torsion subgroup of $P$. The group $G$ just described is a generic torsion-complete $p$-group, and it is a well-known fact $[\mathrm{F}]$ that such groups have the property that we have defined above as being transitive with respect to isotype subgroups.

Example 2. Let $G$ be an arbitrary totally projective $p$-group. Such groups are also known by the titles simply presented and axiom 3 groups. They include all countable $p$-groups and their coproducts. The following theorem of Hill and Megibben implies that every totally projective group is transitive with respect to isotype subgroups.

Recall that $G[p]=\{x \in G: p x=0\}$ and that the $\alpha$ th Ulm invariant of $G$ is the dimension of the vector space $p^{\alpha} G[p] / p^{\alpha+1} G[p]$ over $Z / p Z$. If $H$ is a subgroup of $G$, the dimension of the space $p^{\alpha} G[p] / p^{\alpha} G[p] \cap\left\langle H, p^{\alpha+1} G\right\rangle$ is the $\alpha$ th Ulm invariant of $G$ relative to $H$.

Theorem 1 (Main Theorem, $[\mathrm{HM}]$ ). Let $H$ and $H^{\prime}$ be isotype subgroups of a totally projective group $G$. Suppose that $H$ and $H^{\prime}$ satisfy the conditions:

$\left(1^{\prime}\right) H$ and $H^{\prime}$ have the same Ulm invariants, and

(2) $G / H \simeq G / H^{\prime}$ as valuated groups.

Then there is an automorphism $\pi$ of $G$ that maps $H$ onto $H^{\prime}$. Moreover, if $\phi$ : $G / H \rightarrow G / H^{\prime}$ is a value-preserving isomorphism, there is such an automorphism $\pi$ that induces $\phi$.

The preceding theorem suggests two different refinements of the concept of transitivity. The first of these is to replace condition (1) by the weaker condition $\left(1^{\prime}\right)$ used in the theorem, which does not require a priori that $H$ and $H^{\prime}$ be isomorphic as specified in Definition 1 . We say that the $p$-group $G$ is $U$-transitive (with "U" standing for "Ulm") if any two isotype subgroups $H$ and $H^{\prime}$ that satisfy conditions $\left(1^{\prime}\right)$ and (2) correspond under some automorphism $\pi$ of $G$. The second refinement 
of the notion of transitivity is to strengthen the requirement on the automorphism $\pi$ so that, in accordance with the theorem, it induces a given value-preserving isomorphism $\phi: G / H \rightarrow G / H^{\prime}$. Closely related to the latter refinement is the concept of strongly equivalent subgroups.

Definition 2. Two equivalent subgroups $H$ and $H^{\prime}$ of $G$ are strongly equivalent (although this is not an equivalence relation) if, for any isomorphism $\phi: G / H \rightarrow G / H^{\prime}$ of the quotient groups that preserves the coset valuation, there is an automorphism $\pi$ of the group $G$ that induces $\phi$.

We now combine the two refinements of transitivity mentioned above. The resulting class of groups is the main object of our study.

Definition 3. The $p$-group $G$ is called strongly $U$-transitive if every pair of isotype subgroups $H$ and $H^{\prime}$ are strongly equivalent, provided they satisfy the conditions:

(1') $H$ and $H^{\prime}$ have the same Ulm invariants, and

(2) there exists a value-preserving isomorphism $\phi: G / H \rightarrow G / H^{\prime}$ between the quotient groups.

Remark. Clearly, if $\pi$ is an automorphism of $G$ that induces an isomorphism $\phi$ : $G / H \rightarrow G / H^{\prime}$, then $\pi$ must map $H$ onto $H^{\prime}$. Therefore, $H$ and $H^{\prime}$ are equivalent.

As we have seen in Theorem 1, totally projective groups are strongly U-transitive. An interesting problem is to characterize the $p$-groups that are strongly $\mathrm{U}$-transitive. Using the set-theoretical assumption that $V=L$ (more specifically, using $\diamond$ ), one of the authors has proved in [W] that if $G$ is a $p$-group of cardinality at most $\aleph_{1}$ and if $G$ has countable length (in the sense that $p^{\lambda} G=0$ for some countable ordinal $\lambda$ ), then $G$ is totally projective if and only if $G$ is strongly U-transitive. In the next section we shall establish some closure properties of the class of strongly U-transitive groups.

\section{Properties of strongly U-transitive groups}

The significance of the role of the additional requirement for strong U-transitivity, not present in U-transitivity, is reflected in the proof of the following proposition.

Proposition 1. A summand of a strongly $U$-transitive group is again strongly $U$ transitive.

Proof. Suppose that $G$ is strongly U-transitive, and let $G=A \oplus B$. Suppose that $H$ and $H^{\prime}$ are isotype subgroups of $A$ that satisfy the conditions:

(1') $H$ and $H^{\prime}$ have the same Ulm invariants, and

(2) there is a value-preserving isomorphism $\phi: A / H \rightarrow A / H^{\prime}$.

Since $A$ is a summand of $G$, it is an isotype subgroup of $G$. Therefore, the coset value $\|a+H\|$, for $a \in A$, is the same when computed in $G$ as it is when computed in $A$. Obviously, $\phi$ can be extended to an isomorphism $\phi^{+}$between $G / H$ and $G / H^{\prime}$ by letting $\phi^{+}$be the identity on the complementary summand $B$, and coset values remain preserved under $\phi^{+}$.

Since $G$ is strongly U-transitive, there is an automorphism $\pi$ of $G$ that induces $\phi^{+}$. Consequently, $\pi$ must map $H$ onto $H^{\prime}$, but $\pi$ must also map $A$ onto itself since $\phi^{+}=\phi$ when restricted to $A / H$. We conclude that the restriction of $\pi$ to $A$ is an automorphism of $A$ that induces $\phi$. This demonstrates that $A$ is strongly U-transitive. 
Remark. As we suggested before the statement of Proposition 1, the preceding proof does not work for U-transitivity, nor does the proof stand if we use the concept of transitivity of isotype subgroups defined in Definition 1. The strong versions are needed in both cases.

As we indicated earlier, our results here are based on the proof of the Main Theorem of $[\mathrm{HM}]$, which is quoted above as Theorem 1; in fact, one of our proofs requires a generalization of this theorem, which we will now establish. This result actually generalizes both Theorem 1 and the following vintage extension theorem of one of the authors.

Theorem 0. Let $A$ and $A^{\prime}$ be nice subgroups of $G$ and $G^{\prime}$, respectively, where $G / A$ and $G^{\prime} / A^{\prime}$ are totally projective, and suppose that the Ulm invariants of $G$ relative to $A$ are the same as those of $G^{\prime}$ relative to $A^{\prime}$. If $\rho$ is any height-preserving isomorphism from $A$ onto $A^{\prime}$, then $\rho$ can be extended to an isomorphism from $G$ to $G^{\prime}$.

We are grateful to the referee for pointing out that our original proof of a more technical generalization of Theorem 1 that did not encompass Theorem 0 essentially could be used to prove the theorem that follows, which generalizes both theorems.

Theorem 2. Let $A$ and $A^{\prime}$ be nice subgroups of $G$ and $G^{\prime}$, respectively, where $G / A$ and $G^{\prime} / A^{\prime}$ are totally projective, and let $H$ and $H^{\prime}$ be isotype subgroups of $G$ and $G^{\prime}$. Suppose that $A$ and $H$ and that $A^{\prime}$ and $H^{\prime}$ are compatible subgroups of $G$ and $G^{\prime}$, respectively. Further, suppose that the Ulm invariants of $H$ relative to $A \cap H$ are the same as those of $H^{\prime}$ relative to $A^{\prime} \cap H^{\prime}$. If $\phi: G / H \rightarrow G^{\prime} / H^{\prime}$ is any valuepreserving isomorphism and $\rho: A \rightarrow A^{\prime}$ is any height-preserving isomorphism for which $\rho(a)+H^{\prime}=\phi(a+H)$ for all $a \in A$, then there exists an isomorphism $\pi$ from $G$ to $G^{\prime}$ that simultaneously lifts $\phi$ and extends $\rho$.

Remark. The compatibility hypotheses are redundant in case either $A=0$ or $H=$ $G$, since these two subgroups are always compatible with any subgroup of $G$. In view of this observation, the theorem becomes Theorem 0 if we take $H=G$ and $H^{\prime}=G^{\prime}$. On the other hand, by taking $A=0$ and $A^{\prime}=0$, we obtain Theorem 1 upon setting $G=G^{\prime}$.

Proof. Let $\phi: G / H \rightarrow G^{\prime} / H^{\prime}$ be a value-preserving isomorphism and let $\rho: A \rightarrow A^{\prime}$ be any height-preserving isomorphism that satisfies $\rho(a)+H^{\prime}=\phi(a+H)$ whenever $a \in A$. Assume all the hypotheses of the theorem. Note that $a \in A \cap H$ if and only if $\rho(a) \in A^{\prime} \cap H^{\prime}$, because $\rho(a)+H^{\prime}=\phi(a+H)$.

By mimicking the proof of Theorem 3.2 in [HM], we are able to construct, piece by piece, an isomorphism $\pi: G \rightarrow G^{\prime}$ that extends $\rho$ on $A$. Moreover, we can do this in such a way that $\pi$ induces $\phi$. Indeed, $\phi$ will be our guide throughout the construction of $\pi$. More specifically, the construction of $\pi$ is as follows.

For each $\alpha$, let

$$
\begin{aligned}
p^{\alpha} H[p] & =S_{\alpha} \oplus p^{\alpha+1} H[p] \\
p^{\alpha} H^{\prime}[p] & =S_{\alpha}^{\prime} \oplus p^{\alpha+1} H^{\prime}[p]
\end{aligned}
$$

Then we define

$$
\begin{aligned}
S_{\alpha}(A \cap H) & =\left\{s \in S_{\alpha}:|s+x|>\alpha \text { for some } x \in A \cap H\right\} \\
S_{\alpha}^{\prime}\left(A^{\prime} \cap H^{\prime}\right) & =\left\{s^{\prime} \in S_{\alpha}^{\prime}:\left|s^{\prime}+x^{\prime}\right|>\alpha \text { for some } x^{\prime} \in A^{\prime} \cap H^{\prime}\right\} .
\end{aligned}
$$


The restriction of the isomorphism $\rho: A \rightarrow A^{\prime}$ to an isomorphism between $A \cap H$ and $A^{\prime} \cap H^{\prime}$ can be shown to induce an isomorphism $\mu_{\alpha}: S_{\alpha}(A \cap H) \rightarrow S_{\alpha}^{\prime}\left(A^{\prime} \cap H^{\prime}\right)$ as follows. If $s \in S_{\alpha}$ and $|s+x|>\alpha$ with $x \in A \cap H$, then $p x \in p^{\alpha+2} H$, since $p s=0$ and $H$ is isotype. Set $x^{\prime}=\rho(x)$, and observe that $\left|x^{\prime}\right|=\alpha$ since $|x|=\alpha$ and $\rho$ preserves heights. Moreover, $x^{\prime} \in A^{\prime} \cap H^{\prime}$ since $\rho(A \cap H)=A^{\prime} \cap H^{\prime}$. Also, $\left|p x^{\prime}\right|=|p x|=|p(s+x)| \geq \alpha+2$. Therefore, $p x^{\prime}=p h^{\prime}$, where $h^{\prime} \in p^{\alpha+1} H^{\prime}$. Hence, $x^{\prime}-h^{\prime} \in p^{\alpha} H^{\prime}[p]$ and $h^{\prime}-x^{\prime}=s^{\prime}+t^{\prime}$, where $s^{\prime} \in S_{\alpha}^{\prime}$ and $t^{\prime} \in p^{\alpha+1} H^{\prime}[p]$. Notice that $s^{\prime}+x^{\prime} \in p^{\alpha+1} H^{\prime}$, so $\left|s^{\prime}+x^{\prime}\right|>\alpha$ and $s^{\prime} \in S_{\alpha}^{\prime}\left(A^{\prime} \cap H^{\prime}\right)$. The correspondence $s \rightarrow s^{\prime}$ is the desired isomorphism from $S_{\alpha}(A \cap H)$ onto $S_{\alpha}^{\prime}\left(A^{\prime} \cap H^{\prime}\right)$.

Since the relative Ulm invariants of $H$ with respect to $A \cap H$ are the same as those of $H^{\prime}$ with respect to $A^{\prime} \cap H^{\prime}$, the isomorphism $\mu_{\alpha}: S_{\alpha}(A \cap H) \rightarrow S_{\alpha}^{\prime}\left(A^{\prime} \cap H^{\prime}\right)$ described above can be extended to an isomorphism $\mu_{\alpha}: S_{\alpha} \rightarrow S_{\alpha}^{\prime}$ from $S_{\alpha}$ onto $S_{\alpha}^{\prime}$. Observe that if $s \in S_{\alpha}$ then we have shown that $|s+x|>\alpha$ for $x \in A \cap H$ if and only if $\left|\mu_{\alpha}(s)+\rho(x)\right|>\alpha$. We need to show that this same condition holds for any $x \in A$. In order to do this, we make use of the compatibility of $A$ and $H$ and that of $A^{\prime}$ and $H^{\prime}$. Suppose now that for $s \in S_{\alpha}$ and $a \in A$ we have that $|s+a|>\alpha$. Since $A$ and $H$ are compatible and since $s \in H$, we know that $|s+c|>\alpha$ for some $c \in A \cap H$. From what has gone before, we can conclude that $\left|\mu_{\alpha}(s)+\rho(c)\right|>\alpha$. Since $|s+a|>\alpha$ and $|s+c|>\alpha$, clearly $|a-c|>\alpha$, and therefore $|\rho(a)-\rho(c)|>\alpha$. Hence, $\left|\mu_{\alpha}(s)+\rho(a)\right|>\alpha$. We have shown that $|s+a|>\alpha$ implies that $\left|\mu_{\alpha}(s)+\rho(a)\right|>\alpha$, and symmetry between $G$ and $G^{\prime}$ and their corresponding subgroups yields the implication in the other direction, so we have proved

$$
|s+a|>\alpha \Longleftrightarrow\left|\mu_{\alpha}(s)+\rho(a)\right|>\alpha
$$

whenever $s \in S_{\alpha}$ and $a \in A$.

Assume that $M$ and $N$ are nice subgroups of $G$ and $G^{\prime}$ respectively, and that $\pi: M \rightarrow N$ is an isomorphism that satisfies the following conditions:

(i) $\pi: M \rightarrow N$ is a height-preserving isomorphism that extends $\rho: A \rightarrow A^{\prime}$.

(ii) $\pi(z)+H^{\prime}=\phi(z+H)$ for all $z \in M$.

(iii) For each $\alpha$, if $s \in S_{\alpha}$ and $z \in M,|s+z| \geq \alpha+1$ if and only if $\left|\mu_{\alpha}(s)+\pi(z)\right| \geq$ $\alpha+1$.

It is, of course, crucial that the above conditions are satisfied in the beginning when $M=A, N=A^{\prime}$, and $\pi=\rho$. In this initial case, conditions (i) and (ii) are part of the hypotheses, and condition (iii) is simply $(*)$. Thus the conditions (i)-(iii) are initially satisfied.

We emphasize now that $M$ and $N$ are nice subgroups of $G$ and $G^{\prime}$, respectively, containing $A$ and $A^{\prime}$, and we especially emphasize that $G / A$ and $G^{\prime} / A^{\prime}$ are totally projective. As is well known, nice subgroups of $G / A$ correspond to nice subgroups of $G$. As would be expected, we utilize the axiom 3 characterization of totally projective groups. Recall that this means that a totally projective group has a (distinguished) collection $\mathcal{C}$ of nice subgroups that is closed with respect to group unions (including a vacuous collection) and has the property that any countable subgroup of the group is contained in a member of $\mathcal{C}$ that is also countable. From this description of totally projective groups and by what are now standard arguments in extending maps on nice subgroups of totally projective groups, in order to prove that there is an isomorphism $\pi: G \rightarrow G^{\prime}$ that lifts $\phi$ and extends $\rho$, it suffices to prove the following. For any $x \in G$ that is proper with respect to $M$ and such that $p x \in M$, the isomorphism $\pi: M \rightarrow N$ can be extended, for some $y \in G^{\prime}$, to 
an isomorphism $\pi^{+}:\langle M, x\rangle \rightarrow\langle N, y\rangle$ that continues to satisfy conditions (i)-(iii). In other words, what we need to do is prove, in the context of our hypotheses, that isomorphisms satisfying (i)-(iii) always have local extensions. This step is the very heart of the proof of the theorem, so we will provide details on how this is accomplished. There are two cases. Let $\beta=|x|$.

Case 1. $|p x|>\beta+1$ and $\|x+H\|>\beta+1$.

Case 2. $|p x|=\beta+1$ or $\|x+H\|=\beta+1$.

In each case, we are going to find a companion element $y$ to $x$ with the following five properties.

(a) $|y|=\beta$.

(b) $p y=\pi(p x)$.

(c) $y$ is proper with respect to $N$.

(d) $y+H^{\prime}=\phi(x+H)$.

(e) If $s \in S_{\beta}$ and $z \in M$, then $|s+x+z| \geq \beta+1$ if and only if $\left|\mu_{\beta}(s)+y+\pi(z)\right| \geq$ $\beta+1$.

Then $\pi: M \rightarrow N$ can indeed be extended to $\pi^{+}:\langle M, x\rangle \rightarrow\langle N, y\rangle$ by setting $\pi^{+}(x)=y$, and $\pi^{+}$continues to satisfy conditions (i)-(iii). Finding the desired element $y$ is done as follows.

Case 1. Mindful of property (d), we first choose $x^{\prime} \in G^{\prime}$ so that $\phi(x+H)=$ $x^{\prime}+H^{\prime}$. Since $\phi$ is value preserving, $\left\|x^{\prime}+H^{\prime}\right\|>\beta+1$, and thus we may assume that $\left|x^{\prime}\right|>\beta$. Then by condition (ii) on the map $\pi$, we have that $\phi(p x+H)=$ $\pi(p x)+H^{\prime}=p x^{\prime}+H^{\prime}$, and hence $\pi(p x)-p x^{\prime} \in H^{\prime} \cap p^{\beta+2} G^{\prime}=p^{\beta+2} H^{\prime}$. Therefore, there exists $h^{\prime} \in p^{\beta+1} H^{\prime}$ such that $p h^{\prime}=\pi(p x)-p x^{\prime}$. Notice that $p\left(x^{\prime}+h^{\prime}\right)=\pi(p x)$. If we exchange $x^{\prime}$ for $x^{\prime}+h^{\prime}$, then $x^{\prime}$ satisfies (b) as well as (d).

Now, due to the fact that $\|x+H\|>\beta+1$, there exists $h \in H$ such that $|x+h| \geq \beta+1$. Therefore, $|h|=|x|=\beta$, and also since $|p(x+h)| \geq \beta+2$ and $|p x| \geq \beta+2$, we know that $|p h| \geq \beta+2$. So $p h=p \bar{h}$ for some $\bar{h} \in p^{\beta+1} H$, and thus $\bar{h}-h \in p^{\beta} H[p]$. Write $\bar{h}-h=s+t$, where $s \in S_{\beta}$ and $t \in p^{\beta+1} H[p]$, and we have that $s=\bar{h}-h-t=x-[(x+h)-\bar{h}+t]=x-v$, where $v \in p^{\beta+1} G$. Let $y=x^{\prime}+\mu_{\beta}(s)$, and note that $y$ satisfies conditions (a), (b), and (d). But also since $x$ is proper with respect to $M$ and $v \in p^{\beta+1} G, s=x-v$ is proper with respect to $M$ as well. Then condition (iii) on $\pi$ with $\alpha=\beta$ implies that $\mu_{\beta}(s)$ is proper with respect to $N$, and thus $y$ is also proper with respect to $N$ since $x^{\prime} \in p^{\beta+1} G^{\prime}$. So (c) holds.

In order to establish (e), let $\bar{s} \in S_{\beta}$ and $z \in M$. Then $|\bar{s}+x+z| \geq \beta+1$ if and only if $|\bar{s}+s+z| \geq \beta+1$, since $x=s+v$. The latter is equivalent to the statement $\left|\mu_{\beta}(\bar{s}+s)+\pi(z)\right| \geq \beta+1$ by condition (iii), which holds if and only if $\left|\mu_{\beta}(\bar{s})+y+\pi(z)\right| \geq \beta+1$, since $y=\mu_{\beta}(s)+x^{\prime}$.

Case 2. As in Case 1, let $\phi(x+H)=x^{\prime}+H^{\prime}$, and here we may assume that $\left|x^{\prime}\right| \geq \beta$. Then by (ii), $\pi(p x)-p x^{\prime} \in H^{\prime} \cap p^{\beta+1} G^{\prime}$, so there exists $h^{\prime} \in p^{\beta} H^{\prime}$ such that $p\left(x^{\prime}+h^{\prime}\right)=\pi(p x)$, as above. Set $y=x^{\prime}+h^{\prime}$, and we know that $y$ satisfies properties (b) and (d). But also due to the fact that $y \in p^{\beta} G^{\prime},|y|=\beta$, since in this case either $|p y|=|p x|=\beta+1$ or $|y|<\left\|y+H^{\prime}\right\|=\|x+H\|=\beta+1$. So (a) holds. Now suppose that $y$ is not proper with respect to $N$. Then $|y+\pi(z)| \geq \beta+1$ for some $z \in M$.

Consider the element $\bar{x}=x+z$. We claim that we can exchange the element $x$ for $\bar{x}$, and go back to Case 1. First note that $|x+z| \geq \beta$, since $|z|=|\pi(z)|=|y|=\beta$. But because $x$ is proper with respect to $M,|x+z| \leq \beta$; that is, $|\bar{x}|=\beta$ and $\bar{x}$ is 
proper with respect to $M$. Also, $|p \bar{x}|=|\pi(p \bar{x})|=|p y+\pi(p z)|>\beta+1$. And finally, $\|\bar{x}+H\|=\|\phi(\bar{x}+H)\|=\left\|x^{\prime}+\pi(z)+H^{\prime}\right\|=\left\|y+\pi(z)+H^{\prime}\right\|>|y+\pi(z)| \geq \beta+1$. So if (c) does not hold for $y$, we can return to Case 1 in order to find a companion element $\bar{y}$ to $\bar{x}$.

In order to verify property (e), let $s \in S_{\beta}$ and $z \in M$. If $|s+x+z| \geq \beta+1$ or $\left|\mu_{\beta}(s)+y+\pi(z)\right| \geq \beta+1$, then consider $\bar{x}=x+z$. It turns out that $|\bar{x}|=\beta$, $|p \bar{x}|>\beta+1$, and $\|\bar{x}+H\|>\beta+1$, and thus we may again return to Case 1. Indeed, since $|s|=\left|\mu_{\beta}(s)\right|=\beta$, either $|x+z|=\beta$ or $|y+\pi(z)|=\beta$. In the latter case, $|y|=\beta$ implies $|\pi(z)|=|z| \geq \beta$, so that $|x+z| \geq \beta$. But also $|x+z| \leq|x|=\beta$. And $|p \bar{x}|=|p(s+x+z)|=\left|p\left(\mu_{\beta}(s)+y+\pi(z)\right)\right|$, one of which is greater than $\beta+1$. Finally, $|s+x+z| \geq \beta+1$ clearly implies that $\|\bar{x}+H\|>\beta+1$. If $\left|\mu_{\beta}(s)+y+\pi(z)\right| \geq \beta+1$, then

$$
\begin{aligned}
\|\bar{x}+H\| & =\|\phi(\bar{x}+H)\|=\|\pi(z)+\phi(x+H)\|=\left\|\pi(z)+x^{\prime}+H^{\prime}\right\| \\
& =\left\|\pi(z)+y+H^{\prime}\right\|>\left|\pi(z)+y+\mu_{\beta}(s)\right| \geq \beta+1 .
\end{aligned}
$$

Therefore, we can indeed find $y$ with the desired properties, and the local extension is established. For the reasons given earlier in the proof, relating to the axiom 3 description of totally projective groups, we can now conclude that there is an isomorphism from $G$ to $G^{\prime}$ that simultaneously lifts $\phi$ and extends $\rho$.

Although the next result could be appropriately designated as a corollary of Theorem 2, we prefer to regard it as a lemma since it will be used to prove two subsequent theorems.

Lemma 1. Suppose that $G$ is a p-group and that $\lambda$ is an ordinal for which $G / p^{\lambda} G$ is totally projective. Let $H$ and $H^{\prime}$ be isotype subgroups of $G$ that satisfy conditions (1') and (2). If $p^{\lambda} H$ and $p^{\lambda} H^{\prime}$ are strongly equivalent in $p^{\lambda} G$, then $H$ and $H^{\prime}$ are strongly equivalent in $G$.

Proof. Let $\phi: G / H \rightarrow G / H^{\prime}$ be any isomorphism between $G / H$ and $G / H^{\prime}$ that preserves the coset valuation. This isomorphism induces an isomorphism $\phi_{\lambda}$ : $p^{\lambda} G / p^{\lambda} H \rightarrow p^{\lambda} G / p^{\lambda} H^{\prime}$ that also preserves the coset valuation of these quotient groups computed in $p^{\lambda} G$; this is by virtue of the equalities $p^{\lambda} H=p^{\lambda} G \cap H$ and $p^{\lambda} H^{\prime}=p^{\lambda} G \cap H^{\prime}$ together with the valuated isomorphisms

$$
\begin{aligned}
p^{\lambda} G / p^{\lambda} G \cap H & \simeq p^{\lambda} G+H / H, \\
p^{\lambda} G / p^{\lambda} G \cap H^{\prime} & \simeq p^{\lambda} G+H^{\prime} / H^{\prime} .
\end{aligned}
$$

Since, by hypothesis, $p^{\lambda} H$ and $p^{\lambda} H^{\prime}$ are strongly equivalent in $p^{\lambda} G$, there is an automorphism $\pi_{\lambda}$ of $p^{\lambda} G$ that induces $\phi_{\lambda}$. In particular, $\pi_{\lambda}$ maps $p^{\lambda} H$ onto $p^{\lambda} H^{\prime}$. The desired conclusion now follows from Theorem 2 if we let $\rho=\pi_{\lambda}, G=G^{\prime}$, and $A=p^{\lambda} G=A^{\prime}$; note that every subgroup of $G$ is compatible with $p^{\lambda} G$.

Using the preceding lemma, we can now prove the following theorem. This result can be interpreted to mean, relative to any $\lambda$, that if the top part of $G$ is totally projective and if the bottom part is strongly U-transitive, then $G$, itself, must be strongly U-transitive.

Theorem 3. Suppose that $G$ is a p-group and that $\lambda$ is an ordinal for which $G / p^{\lambda} G$ is totally projective (which is always the case if $\lambda$ is finite). If $p^{\lambda} G$ is strongly $U$ transitive, then $G$ is also strongly $U$-transitive. 
Proof. Suppose that $G$ satisfies the hypotheses of the theorem. Let $H$ and $H^{\prime}$ be isotype subgroups of $G$ that satisfy conditions $\left(1^{\prime}\right)$ and (2), and let $\phi: G / H \rightarrow G / H^{\prime}$ be an arbitrary isomorphism between the quotients $G / H$ and $G / H^{\prime}$ that preserves the coset valuation. It is easy to verify that $p^{\lambda} H$ and $p^{\lambda} H^{\prime}$ are isotype subgroups of $p^{\lambda} G$ that satisfy the same conditions. More precisely, $p^{\lambda} H$ and $p^{\lambda} H^{\prime}$ have the same Ulm invariants, and there is an isomorphism $\phi_{\lambda}: p^{\lambda} G / p^{\lambda} H \rightarrow p^{\lambda} G / p^{\lambda} H^{\prime}$ (induced by $\phi$ ) that preserves the coset values computed in $p^{\lambda} G$. Since, by hypothesis, $p^{\lambda} G$ is strongly U-transitive, the subgroups $p^{\lambda} H$ and $p^{\lambda} H^{\prime}$ must be strongly equivalent in $p^{\lambda} G$. By Lemma $1, G$ is strongly U-transitive.

We have just proved that if $p^{\lambda} G$ is strongly $\mathrm{U}$-transitive, then $G$ is strongly Utransitive provided that $G / p^{\lambda} G$ is totally projective. The next theorem shows that the converse is true whether or not $G / p^{\lambda} G$ is totally projective.

Theorem 4. If $G$ is strongly $U$-transitive, then $p^{\lambda} G$ is also strongly $U$-transitive for any ordinal $\lambda$.

Proof. Assume that $G$ is strongly U-transitive, and let $K$ and $K^{\prime}$ be isotype subgroups of $p^{\lambda} G$ that satisfy:

(a) $K$ and $K^{\prime}$ have the same Ulm invariants, and

(b) there is an isomorphism $\phi_{\lambda}: p^{\lambda} G / K \rightarrow p^{\lambda} G / K^{\prime}$ that preserves the coset values computed in $p^{\lambda} G$.

Let $H$ be maximal in $G$ with respect to $H \cap p^{\lambda} G=K$. Likewise, let $H^{\prime}$ be maximal in $G$ with respect to $H^{\prime} \cap p^{\lambda} G=K^{\prime}$. (The fact that $H$ and $H^{\prime}$ are not unique is of no consequence; any choice will do.) The subgroups $H$ and $H^{\prime}$ are necessarily isotype in $G$. The proof that $H$, for example, is isotype in $G$ is a standard inductive proof that $p^{\alpha} G \cap H=p^{\alpha} H$ for all $\alpha \leq \lambda$. If $\alpha>\lambda$, then $p^{\alpha} G \cap H=p^{\alpha} H$ follows at once from the hypothesis that $p^{\lambda} G \cap H=p^{\lambda} H$ is isotype in $p^{\lambda} G$.

In order to complete the proof of the theorem, it is convenient at this point to consider two cases depending on whether $\lambda$ is a limit ordinal or not. Actually, we will first consider the case $\lambda=1$.

Case 1. $\lambda=1$. In this case, the isotype subgroups $H$ and $H^{\prime}$ are maximal with respect to having $K$ and $K^{\prime}$, respectively, as their intersection with $p G$. Therefore, $H[p] / p H[p] \simeq G[p] / p G[p] \simeq H^{\prime}[p] / p H^{\prime}[p]$, so the 0th Ulm invariant of $H$ is the same as that of $H^{\prime}$. By hypothesis, $p H=K$ and $p H^{\prime}=K^{\prime}$ are known to have the same Ulm invariants, but these are just the higher invariants of $H$ and $H^{\prime}$. Thus, $H$ and $H^{\prime}$ must have the same Ulm invariants.

Recall, for the case $\lambda=1$, that we have an isomorphism $\phi_{1}: p G / p H \rightarrow p G / p H^{\prime}$ that preserves the coset valuation in the group $p G$. The isomorphism $\phi_{1}$ gives rise to an isomorphism $\tilde{\phi}_{1}: p(G / H) \rightarrow p\left(G / H^{\prime}\right)$ through the natural isomorphisms

$$
\begin{aligned}
p G / p H & =p G / p G \cap H \simeq p G+H / H=p(G / H), \\
p G / p H^{\prime} & =p G / p G \cap H^{\prime} \simeq p G+H^{\prime} / H^{\prime}=p\left(G / H^{\prime}\right) .
\end{aligned}
$$

It is easily seen that $\tilde{\phi}_{1}$ preserves coset values computed in $G$, since $\phi_{1}$ preserves coset values computed in $p G$. Since $H$ is maximal in $G$ with respect to $H \cap p G=$ $K$, it quickly follows that the 0th Ulm invariant of $G / H$ is zero; in other words, $(G / H)[p]=p(G / H)[p]$. Now, in view of the fact that $G / H$ and $G / H^{\prime}$ have the same 0th Ulm invariant, the isomorphism $\tilde{\phi}_{1}: p(G / H) \rightarrow p\left(G / H^{\prime}\right)$ can be extended to an isomorphism $\phi: G / H \rightarrow G / H^{\prime}$. The extended isomorphism $\phi$ must necessarily 
preserve the coset values computed in $G$, since its restriction to $\tilde{\phi}_{1}: p(G / H) \rightarrow$ $p\left(G / H^{\prime}\right)$ is value preserving.

Since $G$ is strongly U-transitive, there is an automorphism $\pi$ of $G$ that induces $\phi$. Clearly, $\pi$ restricted to $p G$ induces $\phi_{1}$, so $p G$ is strongly $\mathrm{U}$-transitive. This completes the proof of the theorem for the case $\lambda=1$.

We have shown that $p G$ always inherits strong $\mathrm{U}$-transitivity from $G$. Therefore, by induction so does $p^{n} G$ for every positive integer $n$. It now suffices to prove the theorem for an arbitrary limit ordinal $\lambda$.

Case 2. $\lambda$ is a limit. As in the previous case, we need to prove again that $H$ and $H^{\prime}$ satisfy conditions $\left(1^{\prime}\right)$ and $(2)$. Actually these facts are fairly well known in the case that $\lambda$ is a limit, for the following reasons. We know that $G=\left\langle H, p^{\alpha} G\right\rangle$ for each $\alpha<\lambda$. Since $H$ is isotype, this implies that the $\alpha$ th Ulm invariant of $H$ is the same as the $\alpha$ th Ulm invariant of $G$ for all $\alpha<\lambda$. The same statement can be made about $H^{\prime}$. Hence $H$ and $H^{\prime}$ have the same Ulm invariants up to $\lambda$. But $p^{\lambda} H=K$ and $p^{\lambda} H^{\prime}=K^{\prime}$ have the same Ulm invariants by hypothesis. Since the $(\lambda+\sigma)$ th invariant of $H$ is the $\sigma$ th invariant of $p^{\lambda} H$, for any $H$, it follows that $H$ and $H^{\prime}$ have the same Ulm invariants.

It remains to prove that there exists a value-preserving isomorphism $\phi: G / H \rightarrow$ $G / H^{\prime}$. However, we begin with an isomorphism $\tilde{\phi_{\lambda}}: p^{\lambda} G+H / H \rightarrow p^{\lambda} G+H^{\prime} / H^{\prime}$ induced by $\phi_{\lambda}: p^{\lambda} G / p^{\lambda} H \rightarrow p^{\lambda} G / p^{\lambda} H^{\prime}$. Since $\phi_{\lambda}$ preserves coset values relative to $p^{\lambda} G, \tilde{\phi_{\lambda}}$ must preserve them in $G$. It is well known and, in fact, easily proved that $G / H$ and $G / H^{\prime}$, respectively, are the divisible hulls of $p^{\lambda} G+H / H$ and $p^{\lambda} G+H^{\prime} / H^{\prime}$. Therefore, the isomorphism $\tilde{\phi_{\lambda}}$ can be extended to an isomorphism $\phi: G / H \rightarrow$ $G / H^{\prime}$. Finally, we observe that $\|g+H\| \geq \lambda+1$ if and only if $g+H \in p^{\lambda} G+H / H=$ domain $\tilde{\phi_{\lambda}}$, whereas $\|g+H\|=\lambda$ if $g+H \notin \tilde{p}^{\lambda} G+H / H$. The corresponding statements are true for $H^{\prime}$ and the range of $\tilde{\phi}_{\lambda}$. Therefore, the extension $\phi$ : $G / H \rightarrow G / H^{\prime}$ preserves values.

As in the case $\lambda=1$, we let $\pi$ be an automorphism of $G$ that induces $\phi$. The restriction of $\pi$ to $p^{\lambda} G$ is the desired automorphism of $p^{\lambda} G$ that induces $\phi_{\lambda}$. This completes the proof that $p^{\lambda} G$ is strongly U-transitive.

\section{4. $C_{\Omega}$-Groups ARE STRONGLY $\aleph_{0}$-TRANSitive}

By Ulm's classical theorem, we know that conditions (1) and $\left(1^{\prime}\right)$ are equivalent in case the subgroups $H$ and $H^{\prime}$ are countable. We want to consider now a wide class of groups that are transitive, actually strongly transitive, with respect to countable isotype subgroups.

Definition 4. An abelian $p$-group $G$ is (strongly) $\aleph_{0}$-transitive if any two countable isotype subgroups $H$ and $H^{\prime}$ are (strongly) equivalent in $G$ whenever they satisfy conditions (1) and (2).

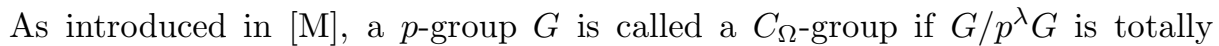
projective for each countable ordinal $\lambda$. Also, recall that the totally projective groups of length not exceeding $\omega_{1}$ are precisely the groups that can be written as direct sums of countable $p$-groups. Isotype subgroups of those groups are always $C_{\Omega}$-groups, and, as was pointed out in $[\mathrm{HM}]$, these groups have the same order of structural complexity as $p$-groups in general. In view of this fact, the following result may be surprising.

Theorem 5. All $C_{\Omega}$-groups are strongly $\aleph_{0}$-transitive. 
Proof. Using the lemma of the previous section, we can prove slightly more. Suppose that $G$ is a $C_{\Omega}$-group and that $H$ and $H^{\prime}$ are isotype subgroups having countable length. Note that any isotype subgroup of $G$ that has countable length must have trivial intersection with $p^{\lambda} G$ for some countable ordinal $\lambda$. Choose a countable

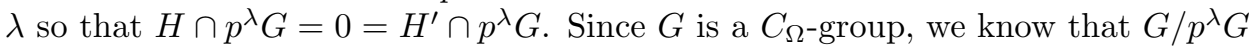
is totally projective. Therefore, if $H$ and $H^{\prime}$ satisfy conditions (1) and (2), we can apply Lemma 1 and conclude that $H$ and $H^{\prime}$ are strongly equivalent. Thus any $C_{\Omega}$-group is strongly $\aleph_{0}$-transitive.

\section{REFERENCES}

[F] L. Fuchs, Infinite Abelian Groups, Volumes I \& II, Academic Press, New York, 1970 and 1973. MR 41:333; MR 50:2362

[H] P. Hill, "On Transitive and Fully Transitive Primary Groups," Proc. Amer. Math. Soc. 22 (1969), 414-417. MR 42:4630.

[HM] P. Hill and C. Megibben, "On the Theory and Classification of Abelian p-Groups," Math. Z. 190 (1985), 17-38. MR 86k:20049

[K] I. Kaplansky, Infinite Abelian Groups, University of Michigan Press, Ann Arbor (1954). MR 16:444g

[M] C. Megibben, "A Generalization of the Classical Theory of Primary Groups," Tôhoku Math. Journ. 22 (1970), 347-356. MR 45:3561

[W] J. K. West, "A $\diamond$-Characterization of Some Small Totally Projective Groups," Lecture Notes in Pure and Applied Math. 182, Marcel Dekker, Inc., New York (1996), 393-401. CMP 97:03

Department of Mathematics, Auburn University, Alabama 36849

Department of Natural Sciences, Colby-Sawyer College, New london, New HampSHIRE 03257 